\title{
HABILIDADES SOCIOEMOCIONALES: ALUMNOS CON DISCAPACIDAD VISUAL EN UN AULA INCLUSIVA DE EDUCACIÓN INFANTIL
}

\author{
Wendy Itzameny Cahum González \\ Estudiante de la maestría en Ciencias de la Educación \\ Instituto de Ciencias Sociales y Humanidades \\ Universidad Autónoma del Estado de Hidalgo, México \\ wendy_cahum@ueah.edu.mx \\ Maricela Zuñiga Rodríguez \\ Profesora investigadora del Área Ciencias de la Educación \\ Instituto de Ciencias Sociales y Humanidades \\ Universidad Autónoma del Estado de Hidalgo, México \\ innomary@hotmail.com
}

Recepción Artículo: 04 septiembre 2021 Admisión Evaluación: 04 septiembre 2021 Informe Evaluador 1: 12 septiembre 2021 Informe Evaluador 2: 16 septiembre 2021 Aprobación Publicación: 19 septiembre 2021

\section{RESUMEN}

La educación socioemocional en niños y niñas con discapacidad visual es un derecho humano, cuyo objetivo es garantizar esta educación de calidad en igualdad de oportunidades con una perspectiva de equidad. En este trabajo se evidencia el avance del análisis las habilidades socioemocionales en alumnos con discapacidad visual en un aula inclusiva de educación infantil, a partir de sus concepciones sobre la educación socioemocional, la relación de las dimensiones socioemocionales y su contrastación con las habilidades socioemocionales presentes en el aula inclusiva, desde la nueva realidad pandémica de enseñanza que actualmente se desarrolla a causa de la emergencia sanitaria del COVID-19, para ello se trabajan referentes teóricos encaminados a las habilidades socioemocionales.

Palabras clave: educación socioemocional; habilidades socioemocionales; inclusión educativa; aula inclusiva; discapacidad visual.

\section{ABSTRACT \\ Social-emotional skills: students with visual impairment in an inclusive early childhood edu- cation classroom. Socio-emotional education in boys and girls with visual disabilities is a human right, the objective of which is to guarantee this quality education with equal opportunities with a perspective of equity. The present objective is to present the partial advances of the research on the analysis of socio-emotional skills in students with visual disabilities in an inclusive preschool classroom, based on their conceptions about socio- emotional education, the relationship of socio-emotional dimensions and their contrast. With the socio-emotional}


skills present in the inclusive classroom, from the new pandemic reality of teaching that is currently developing due to the health emergency of COVID-19, for this, theoretical references aimed at socio-emotional skills are being worked on.

Keywords: socio-emotional education; socio-emotional skills; educational inclusion; inclusive classroom; visual disability.

\section{INTRODUCCIÓN}

La nueva realidad por pandemia COVID-19 ha originado fuertes impactos psicológicos en la colectividad educativa y la sociedad en forma general. La suspensión y cierre de las instituciones educativas, la necesidad y emergencia del distanciamiento físico, la pérdida de seres queridos, la transición de la forma de trabajo y el impedimento de utilizar los métodos de enseñanza-aprendizaje tradicionales han generado presión, estrés, ansiedad y depresión; afectando en específico a los docentes, al alumno y sus familias, esto fundamentado por la UNESCO en el Informe de Promoción del Bienestar Socioemocional de los Niños y los Jóvenes Durante las Crisis (2020).

Bisquerra explica que la Educación Emocional es un proceso educativo, continuo y permanente, que pretende potenciar el desarrollo emocional como complemento indispensable del desarrollo cognitivo, constituyendo ambos los elementos esenciales del desarrollo de la personalidad integral. Para ello se propone el desarrollo de conocimientos y habilidades sobre las emociones con el objetivo de capacitar al individuo para afrontar mejor Ios retos que se le plantean en su vida cotidiana. Todo con la finalidad aumentar el bienestar personal y social, (Bisquerra, 2003, pág. 243).

El contexto familiar, social, escolar, e histórico en donde se desenvuelve un niño con discapacidad visual desde su nacimiento, por algún accidento y/o enfermedad, permean de manera sustancial en su desarrollo. Lo anterior, no debe pasar desapercibido, dado que los contextos en que habitan los niños y niñas determinan su experiencia, forma de aprendizaje, cultura, comportamiento e ideología. Desarrollar y fomentar en los seres humanos habilidades de enseñanza-aprendizaje socioemocional ayuda a que las situaciones extremas que generan respuestas emocionales negativas estresantes se aborden con calma y con respuestas emocionales equilibradas y ecuánimes. Cabe resaltar que estas competencias permiten mejorar y fortalecer el pensamiento crítico para tomar decisiones mejor informadas en la vida, (UNESCO, 2020)

El docente que coexiste con la discapacidad visual en el aula inclusiva, atiende a un grupo heterogéneo y el discernimiento de los niños en esta nueva realidad encarna un reto; el docente se enfrente al desafío tecnológico-personal e incluso se pone al descubierto el autoanalizase y reconocerse a sí mismo, en torno a su ideología, practicas, costumbre y concepción, que los distingue y define a su persona proyectándose a su labor docente, (Gifre Monreal \& Guitart, 2013).

Por ello, es menester que el docente se percate que la población de niños con discapacidad visual que están bajo su tutela, forman parte de un sin número de sistemas e influencias ambientales en las que se ven inmersos desde su nacimiento.

La educación y en específico las habilidades socioemocionales para estos niños no puede ser generalizada, cada uno de ellos, tiene un ritmo diferente aun cuando forme parte de un grupo de niñas y niños con la misma discapacidad visual, por lo cual al implementar mecanismos de apoyo al proceso de enseñanza-aprendizaje, hay que considerar si la familia cuenta con los recursos o materiales para el trabajo desde casa, (Pérez \& Arredondo, 2020)

\section{OBJETIVOS DE LA INVESTIGACIÓN}

\section{Objetivo general}

Investigar el desarrollo de las habilidades socioemocionales que poseen los infantes, como se desarrollan, la forma de compartir, las diferencias de los alumnos con y sin discapacidad visual en aula inclusiva de educación infantil. 


\section{Objetivos específicos}

- Que proceso metodológico utiliza que el docente para desarrollar las habilidades socioemocionales en los alumnos con y sin discapacidad visual en un aula de educación infantil inclusivas.

- Comparar e integrar las habilidades socioemocionales, que comparten o difieren los alumnos con y sin discapacidad visual, en un aula de un educación infantil inclusivo.

- Identificar el impacto de las habilidades socioemocionales que la educadora en educación infantil ha favorecido en los alumnos con y sin discapacidad visual.

\section{MUESTRA Y/O PARTICIPANTES}

La escuela Educación infantil Garabatos, se ubica en la zona del municipio de Tulancingo de Bravo en el estado de Hidalgo, México, la institución es dependencia pública, perteneciente a la Secretaria de Educación Pública (SEP), dentro del nivel educativo básico educación infantil general; cabe hacer mención que por las condiciones de aislamiento pandémico por COVID-19, las clases son de forma virtual, sin embargo, es importante contextualizar la escuela.

La escuela se localiza en un medio urbano, cuenta con todos los servicios públicos, el edificio es de una sola planta consta de 9 aulas de 1a a 3º; una dirección, bodega, cancha deportiva, patio cívico y comedor con cocina integrada.

El contexto social donde se ubica la escuela es considerado violento, existen pandillas y problemas de drogadicción que limitan la recreación familiar, algunos alumnos provienen de este tipo de ambientes, que impacta en su desarrollo social e integral. El nivel socioeconómico que ostenta la población es bajo, realizan oficios de albañilería, plomería, mecánica y costura como medio de sustento, la actividad principal es el comercio informal, las familias de algunos niños y niñas son migrantes de comunidades aledañas, quienes presentan problemas de preparación profesional con un nivel escolar de educación básica o media superior. Existen problemas de desintegración familiar, que tiene como consecuencia familias disfuncionales, donde está presente solo la figura de mamá, papá 0 abuelitos.

La población estudiantil se compone de 3 grupos de $1^{0}$ a $3^{0}$ con tres grados por nivel, consta de un total de 143 niños y niñas, el número de estos oscila entre 25 y 30 por grupo. Los sujetos de estudio son alumnos con y sin discapacidad visual de 1 ro y 2 do grado de una educación infantil inclusivo.

\section{METODOLOGíA}

Esta investigación se posiciona en el paradigma interpretativo cualitativo de estudio de caso desde una posición interpretativa y hermenéutica, con una dimensión naturalista cualitativa y subjetiva, cuyo objetivo es comprender e interpretar la realidad educativa, Ios significados de los sujetos de estudio, las percepciones, intenciones y acciones dentro de un contexto y tiempo determinado (Sánchez, 2013).

Las herramientas de investigación que se utilizarán para recoger los datos será la entrevista semiestructurada y la observación no participantes, instrumentos que responden a los objetivos específicos de la investigación. La entrevista semiestructurada se encuentra en proceso de revisión por parte del comité tutorial, contempla preguntas de análisis y reflexión para que las educadoras expliquen los aspectos didácticos y las prácticas de enseñanza socioemocional. Se ha estructurado en dimensiones y categorías de análisis como: discapacidad visual, habilidades socioemocionales, inclusión y sistemas ecológicos, estos contemplan aspectos didácticos y las estrategias de enseñanza y aprendizaje en el área socioemocional.

La observación no participante es una técnica de investigación que consiste en la recolección de información desde una postura totalmente alejada y sin involucramiento alguno por parte de la investigadora para comprender los diferentes procesos vividos en el aula inclusiva de educación infantil.

\section{RESULTADOS ALCANZADOS}

Este apartado se enfoca en la descripción del tipo de investigación realizada, así como la descripción de las 
fuentes utilizadas. Así mismo, se presenta la forma en que fue procesada la información obtenida con el objetivo de dar a conocer la técnica de investigación que permitió llegar a los objetivos inicialmente planteados. El documento se fundamenta en la metodología documental-descriptiva; lo que implico el análisis de documentos científicos, en este sentido, tanto lo personal como lo subjetivo hacen que el elemento cualitativo se vuelva característico en este tipo de investigaciones, Tena y Rivas-Torres, (1998).

Se base en un diseño no experimental, ya que el objetivo principal de este modelo es el de observar los fenómenos tal y como se dan en su entorno natural y después analizarlos. En esta investigación no se han manipulado las variables, se ha delimitado a la revisión, descripción e interpretación de la información adquirida, por tanto, lo personal como lo subjetivo hacen el elemento cualitativo se vuelva característico en este documento.

En este sentido, se proponen las siguientes estrategias para el desarrollo de las habilidades socioemocionales en niños con discapacidad visual, como estrategia para la enseñanza-aprendizaje por confinamiento de la pandemia por COVID-19.

Los niños, en México, están en sus hogares, por tanto es trascendental que los padres y/o la familia así como los docentes forjen relaciones seguras y de confianza con todos ellos para ayudarlos a entender esta nueva normalidad, adaptarse a ella y progresar.

Identificar, conocer y distinguir los comportamientos del niño con discapacidad visual, ya que el comportamiento es su forma de comunicación, es decir su lenguaje.

Los niños con discapacidad visual y sus padres necesitan la escucha y la empatía del docente como guía.

Estar atentos a la manifestación de posibles señales negativas como el aislamiento, la agresividad, el silencio, el miedo o cualquier manifestación anormal a su comportamiento habitual. Todas ellas indican que los niños necesitan saber que la persona más allegada a él y con quien se basa e inicia el sistema ecológico está presente, con el objetivo de apoyarlos, ayudarlos, acompañarlos y en este sentido desarrollen y fortalezcan sus habilidades socioemocionales trayendo como consecuencia la generación de confianza en los sistemas ecológicos que forman parte de su vida cotidiana.

Organizar reuniones virtuales abordando las zonas de desarrollo próximo para toda la comunidad educativa donde se planteen temas como los sentimientos, las emociones, las habilidades sociales, los sentimientos negativos, las agresiones, el desarrollo de relaciones con niños y familias, o el acoso escolar.

La capacidad de ser innovadores y creativos en el currículo para el desarrollo de habilidades socioemocionales será lo que en el futuro diferencie de un buen proceso de enseñanza-aprendizaje virtual. Los cambios por contingencia pandémica por COVID-19 están sujetos a la innovación curricular y pedagógica, por lo cual la comunidad educativa será aquella que imagine, invente y descubra cómo desarrollar y fortalecer las habilidades socioemocionales en los niños con discapacidad visual en este nuevo contexto de vida.

\section{DISCUSIÓN}

Partiendo de la necesidad de mantener la continuidad en el desarrollo de las habilidades socioemocionales en niños con discapacidad visual, como estrategia para la enseñanza-aprendizaje por confinamiento de la pandemia por COVID-19, surge la importancia de analizar estudios sobre la sensibilidad y armonía para con los estados emocionales de los niños desde el mismo nacimiento, que influirán en su desarrollo y capacidad de ajuste emocional.

Elia López Cassa, en el año 2005, expone que las emociones están presentes en la vida humana desde el nacimiento hasta la muerte, debido al proceso de construcción de la personalidad e interacción social, marcando el cómo intervienen las emociones para poder evolucionar: en el desarrollo de la comunicación, conocimiento social, el procesamiento de la información, el apego, el desarrollo moral, (López Lassá, 2007).

Karl Rogers, pone mayor atención a las emociones; el tipo de afecto que los niños establecen con relación a la madre y el padre, son base en el futuro con sus relaciones sociales, esto motivado en la teoría Humanista, (Rogers, 2011). En este sentido, cualquier crisis, desde desastres naturales, guerras y pandemias como la que se 
vive hoy en día por el COVID-19, trae como consecuencia respuestas emocionales negativas como: pánico, asco, enfado, estrés, ira, agresividad, ansiedad, rabia, miedo, entre otras.

Desarrollar y fomentar en los seres humanos habilidades de enseñanza-aprendizaje socioemocional ayuda a que las situaciones extremas que generan respuestas emocionales negativas estresantes se aborden con calma y con respuestas emocionales equilibradas y ecuánimes. Cabe resaltar que estas competencias permiten mejorar y fortalecer el pensamiento crítico para tomar decisiones mejor informadas en la vida, (UNESCO, 2020)

El docente que convive con la discapacidad visual en el aula virtual, atiende a un grupo heterogéneo y la percepción de los niños en esta nueva realidad encarna un reto; el docente se enfrente al desafío tecnológico-personal e incluso se pone al descubierto el autoanalizase y reconocerse a sí mismo, en torno a su ideología, practicas, costumbre y concepción, que los distingue y define a su persona proyectándose a su labor docente, (Gifre Monreal \& Guitart, 2013). Por ello, es menester que el docente se percate que la población de niños con discapacidad visual que están bajo su tutela, forman parte de un sin número de sistemas e influencias ambientales en las que se ven inmersos desde su nacimiento.

Por ende, la educación para estos niños no puede ser generalizada, cada uno de ellos, tiene un ritmo diferente aun cuando forme parte de un grupo de niñas y niños con la misma discapacidad visual, por lo cual al implementar mecanismos de apoyo al proceso de enseñanza-aprendizaje, hay que considerar si la familia cuenta con los recursos o materiales para el trabajo desde casa, (Pérez \& Arredondo, 2020)

El docente se auxiliará con las características de los niños; de la edad, el contexto en el que viven, lo que están atravesando por esta invidencia o videncia; para así poder ofrecer herramientas, oportunidades, guía, motivación y apoyo socioemocional adecuado; generando empatía, autoconocimiento y autorregulación.

La familia, es otro factor a considerar, ya que puede estar constituida por los padres, hijos, y cualquier otra persona que pertenezca a ella como los abuelos, tíos, sobrinos, primos; esta estructura en la actualidad es difícil de mantener, debido al cambio de roles, las necesidades que se presentan como la económica, la vivienda, la salud y la educación. Ahora bien, a lo que refiere a los padres constituyen el primer escalón y la base de socialización del niño con discapacidad visual; siendo la madre, la guía e introductora en el sistema emocional-social madre-hijo (Hidalgo Carrasco, 2010). Por ende, al tener un niño con discapacidad visual es una causa estresante para la familia, es decir viven emociones negativas, que serán enfrentadas por los padres convirtiendo este vínculo en un factor de unión para confrontar las demandas de la condición de vida que tienen y poner a prueba las habilidades socioemocionales.

Cabe destacar que tanto en educación presencial como la virtual, tiene mucho que ver las actitudes de los padres con respecto a la educación y a la escuela del hijo; estas tienen efectos trascendentales sobre el niño. Si los padres no desean profundizar o conocer la discapacidad visual, no apreciaran el aprendizaje del niño, por tanto el niño con discapacidad no lo hará. Si los padres poseen actitudes positivas de fomentar la superación, la independencia, la autonomía; por consecuencia los alumnos con discapacidad mostrarán mejores niveles de adaptación y ajuste social (Hidalgo Carrasco, 2010). El confinamiento pandémico por COVID-19 exige a la comunidad educativa la preparación de los niños con discapacidad visual en el proceso de descubrimiento y desarrollo de sus habilidades socioemocionales dormidas dentro de su poder personal.

\section{CONCLUSIONES}

Los docentes, los alumnos y la familia han tenido que adaptarse a condiciones retadoras como es el trabajar en línea, preparar clases con tecnologías no usadas ni dominadas, a enviar evidencias de trabajo que antes no se había hecho a toda hora del día y de la noche, las clases ya no son exclusivas para los niños de un grupo 0 un grado determinado, son para toda la familia en sus casas: mamás, papás, hermanos, abuelos en algunos casos; de manera continua la comunidad educativa fue supervisada no solo por los directores de las escuelas, también por supervisores y demás autoridades que tenían acceso para entrar y salir de las clases virtuales. Todas estas condiciones han generado situaciones de crisis emocional a la comunidad educativa, haciendo énfasis en los alumnos con discapacidad visual. 
El confinamiento pandémico por COVID-19 exige a la comunidad educativa la preparación de los niños con discapacidad visual en el proceso de descubrimiento y desarrollo de sus habilidades socioemocionales dormidas dentro de su poder personal; en este sentido el rol de docentes y los padres de familia deben ser capaces de acompañarlos y apoyarlos de manera auténtica, creando y siendo parte de sus sistemas ambientales sanos de enseñanza-aprendizaje que les permitan ser personas sanas en lo físico, fisiológico, emocional y social; individuos capaces de ser resilientes en futuras situaciones adversas como la actual, generando sentimientos positivos emanados de ese desarrollo socioemocional.

Hay que enfatizar que el trabajo del docente y los padres de familia debe ser el crear escenarios favorables para que sus alumnos/hijos con y sin discapacidad visual, en este proceso educativo, puedan descubrir y desarrollar sus habilidades socioemocionales, su poder interior y las distintas formas en que se enfrenta a él mismo y a los otros. Tener conciencia de ello está ligado al desarrollo como sociedad, como personas y al desarrollo humano personal.

Las condiciones favorables para educar y desarrollar las habilidades socioemocionales de los niños con discapacidad visual, implican el trabajo personal del docente, los padres, la familia y el mismo alumno en un ejercicio permanente, consistente y constante de responsabilidad individual y colectiva en el manejo de sus dimensiones y habilidades socioemocionales, logrando el aliciente del crecimiento personal de estos alumnos.

\section{REFERENCIAS BIBLIOGRÁFICA}

Bisquerra, R. (2003). La Educación Emocional y Competencias Básicas para la vida. Revista de Investigación Educativa., 21(1), 7-43.

Gifre Monreal, M., \& Guitart, M. (2013). Consideraciones educativas de la perspectiva ecológica de Urie Bronferbrenner. Contextos Educativos. Revista de Educación, 15(79-92), 79-92. Obtenido de http://dx.doi.org/10.18172/con.656

Hidalgo Carrasco, R. E. (2010). Aspecto psicologicos de los padres de los niños con deficiencia visual. En Guia de ajuste y orientacione emocional para padres de niños con deficiencia visual, en condicione de vulnerabilidad, en el Insituto Especial de Invidentes y Sordos de Azuay (págs. 48-78). Cuenca-Ecuador: Universidad Politécnica Salesiana.

López Lassá, E. (2007). Educación Emocional. Programa para 3-6 años. W. Kluwer, Ed.

Pérez, E. P., \& Arredondo, E. A. (2020). Las personas en situación discapacidad en tiempos de COVID-19. Bajo la Lupa. Chiapas, México: Amor sin Fronteras. A. C.

Rogers, C. (2011). El proceso de convertirse en persona. Madrid: Paidós Ibé-rica.

UNESCO. (2020). Promoción de Ibienestar socioemocional de los niños y los jóvenes durante las crisis. Paris, Francia: Organización de las Naciones Unidas para la Educación, la Ciencia y la Cultura (UNESCO) . 